\title{
Stabilization Analysis for the Switched Large-Scale Discrete-Time Systems via the State-Driven Switching
}

\author{
Chi-Jo Wang and Juing-Shian Chiou \\ Department of Electrical Engineering, Southern Taiwan University of Science and Technology, Tainan, Taiwan \\ Correspondence should be addressed to Juing-Shian Chiou; jschiou@mail.stust.edu.tw
}

Received 15 February 2013; Accepted 7 April 2013

Academic Editor: Her-Terng Yau

Copyright ( 2013 C.-J. Wang and J.-S. Chiou. This is an open access article distributed under the Creative Commons Attribution License, which permits unrestricted use, distribution, and reproduction in any medium, provided the original work is properly cited.

A criterion of stabilization for the switched large-scale discrete-time system is deduced by employing state-driven switching method and the Lyapunov stability theorem. This particular method especially can be applied to cases when all individual subsystems are unstable.

\section{Introduction}

Recently, switched systems have attracted great research attention in academic and industrial applications. They also have been widely and successfully applied to a variety of industrial processes such as the automotive industry, chemical procedure control systems, navigation systems, automobile speed change systems, aircraft control systems, air traffic control, and many other fields [1-7]. Switched systems are a class of hybrid dynamical systems consisting of several continuous-time or discrete-time subsystems and a rule that orchestrates the switching sequences among them. In the study of switched systems, most of the works have been centralized on the problem of stability; see, for example, [8-17] and the references cited therein. Two important methods are used to construct the switching law for the stability analysis of the switched systems. One is the state-driven switching strategy [8-13], and the other is the time-driven switching strategy [14-17]. The time-driven switching method is based on the concept of a dwell time. If there exists at least an individual stable subsystem, then the switched system is stable with appropriate dwell time switching law. But in many applications, all individual subsystems are unstable. Whereas for systems with the state-driven switching strategy, there have been many choices of switching strategy to make the whole system stable even when all individual subsystems are unstable.

Recently, some stability conditions and stabilization approaches have been proposed for the switched discrete-time systems. Reference [18] studied the quadratic stabilization of discrete-time switched linear systems when a designed switching rule is imposed upon the feedback controller of subsystems and studied quadratic stabilization of switched system with norm-bounded time-varying uncertainties. The event-driven scheduling strategy for constructing switching law to stabilize the switched system is presented in [19]. Reference [20] studied stability property for the switched systems which are composed of a continuous-time LTI subsystem and a discrete-time LTI subsystem, when the two subsystems are Hurwitz and Schur stable, respectively. It was shown that if the subsystem matrices are symmetric, then a common Lyapunov function exists for these two subsystems and that the switched system is exponentially stable under arbitrary switching. There exists a switched quadratic Lyapunov function to check asymptotic stability of the switched discrete-time system in [21]. References [22, 23] studied robust stability analysis and control synthesis of uncertain switched systems. Reference [24] has shown that to achieve controllability for a switched linear system, it is sufficient to use cyclic and synchronous switching paths and constant control laws.

Large-scale systems, the mathematical models of many physical and engineering systems, are frequently of high dimension or possess interacting dynamic phenomena. Such systems consist of a number of interdependent subsystems which serve particular functions, share resources, and are governed by a set of interrelated goals and constraints $[25,26]$. 
In real systems, the large-scale systems include electric power systems, nuclear reactors, aerospace systems, large electric networks, economic systems, process control systems, chemical and petroleum industries, different types of societal systems, and ecological systems. Recently, many approaches have been used to investigate the stability and stabilization of large-scale systems [27-33].

As we have indicated, very few research articles have ever discussed the stabilization of the switched large-scale discrete-time system. Therefore, we will investigate the stabilization analysis of this new class of switched large-scale discrete-time systems. In this paper, we will present novel and general approaches by Lyapunov stability theorem to construct a state-driven switching strategy such that the switched large-scale discrete-time system is asymptotically stable. Particularly, this method can be applied to cases when all individual subsystems are unstable.

Notation. The following notations will be used throughout the paper: $\lambda(A)$ stands for the eigenvalues of matrix $A, A_{\bar{\lambda}}=$ $\lambda_{\text {max }}(A)\left(A_{\underline{\lambda}}=\lambda_{\text {min }}(A)\right)$ means the maximum (minimum) eigenvalue in matrix $A,\|A\|$ denotes the norm of matrix $A$, that is, $\|A\|=\operatorname{Max}\left[\lambda\left(A^{T} A\right)\right]^{1 / 2}, A^{T}$ is the transpose of matrix $A$, and $\operatorname{diag}\{\cdots\}$ denotes a block-diagonal matrix.

\section{System Description and Problem Statement}

Consider the switched large-scale discrete-time systems with $r$ individual systems and each individual system composed of $N$ interconnected subsystems:

$$
x_{i}(k+1)=A_{\sigma(x(k))_{i i}} x_{i}(k)+\sum_{\substack{j \neq i \\ j=1}}^{N} A_{\sigma(x(k))_{i j}} x_{j}(k),
$$

where $x_{i}(k) \in R^{n_{i}}$ is the state vectors of the $i$ th subsystem, $i=1,2, \ldots, N, n=\sum_{i=1}^{N} n_{i}, A_{\sigma(x(k))_{i i}}$ and $A_{\sigma(x(k))_{i j}}$ are some constant matrices of compatible dimensions, $x(k)=$ $\left[\begin{array}{llll}x_{1}^{T}(k) & x_{2}^{T}(k) & \cdots & x_{N}^{T}(k)\end{array}\right]^{T} \in R^{n}, \sigma(x(k)): R^{n} \rightarrow\{1,2, \ldots$, $r\}$ is a piecewise constant scalar function of state, called a switch signal; that is, $\sigma(x(k))=l$ implies the subsystem matrices $A_{l_{i i}}$ and $A_{l_{i j}}, l \in\{1,2, \ldots, r\}$. Essentially, the switched discrete-time system (1) can be described as follows.

Individual system $l$ :

$$
x_{i}(k+1)=A_{l_{i i}} x_{i}(k)+\sum_{\substack{j \neq i \\ j=1}}^{N} A_{l_{i j}} x_{j}(k)
$$

For the stabilization of switched large-scale discrete-time system (2), some helpful lemmas are given subsequently.

Lemma 1 (see [34]). For any matrices $A$ and $B$ with appropriate dimensions, one has

$$
A^{T} B+B^{T} A \leq \gamma A^{T} A+\frac{1}{\gamma} B^{T} B
$$

for any constant $\gamma>0$.
Lemma 2. For any matrices $A_{1}, A_{2}, \ldots, A_{N}$ with the same dimensions, the following inequality holds for any positive constant $\varepsilon$ :

$$
\begin{aligned}
\left(\sum_{i=1}^{N} A_{i}\right)^{T}\left(\sum_{i=1}^{N} A_{i}\right) \\
\leq(1+\varepsilon) A_{1}^{T} A_{1}+\left(1+\varepsilon^{-1}\right)(1+\varepsilon) A_{2}^{T} A_{2} \\
\quad+\left(1+\varepsilon^{-1}\right)^{2}(1+\varepsilon) A_{3}^{T} A_{3} \\
\quad+\cdots+\left(1+\varepsilon^{-1}\right)^{N-2}(1+\varepsilon) A_{N-1}^{T} A_{N-1} \\
\quad+\left(1+\varepsilon^{-1}\right)^{N-1} A_{N}^{T} A_{N} .
\end{aligned}
$$

Proof. For a positive constant $\varepsilon, A$ and $B$ have the same dimension, such that

$$
(A+B)^{T}(A+B) \leq(1+\varepsilon) A^{T} A+\left(1+\varepsilon^{-1}\right) B^{T} B .
$$

In view of inequality (5), we have

$$
\begin{aligned}
& \left(\sum_{i=1}^{N} A_{i}\right)^{T}\left(\sum_{i=1}^{N} A_{i}\right) \\
& \leq(1+\varepsilon) A_{1}^{T} A_{1}+\left(1+\varepsilon^{-1}\right)\left(\sum_{i=2}^{N} A_{i}\right)^{T}\left(\sum_{i=2}^{N} A_{i}\right) \\
& \leq(1+\varepsilon) A_{1}^{T} A_{1}+(1+\varepsilon)\left(1+\varepsilon^{-1}\right) A_{2}^{T} A_{2} \\
& +\left(1+\varepsilon^{-1}\right)^{2}\left(\sum_{i=3}^{N} A_{i}\right)^{T}\left(\sum_{i=3}^{N} A_{i}\right) \\
& \leq(1+\varepsilon) A_{1}^{T} A_{1}+(1+\varepsilon)\left(1+\varepsilon^{-1}\right) A_{2}^{T} A_{2} \\
& +\cdots(1+\varepsilon)\left(1+\varepsilon^{-1}\right)^{N-2} A_{N-1}^{T} A_{N-1} \\
& +\left(1+\varepsilon^{-1}\right)^{N-1} A_{N}^{T} A_{N} \cdot
\end{aligned}
$$

Lemma 3. Let $\alpha_{l}=1 /\left(1+\varepsilon^{-1}\right)^{l-1}(1+\varepsilon), l=1,2, \ldots, r-1$, and $\alpha_{r}=1 /\left(1+\varepsilon^{-1}\right)^{r-1}$; then $\alpha_{l} \in[0,1](1 \leq l \leq r)$ and $\sum_{l=1}^{r} \alpha_{l}=1$ for any positive constant $\varepsilon$.

Proof. Obviously, if there exists a positive constant $\varepsilon$ then $0<$ $\alpha_{l}<1, l=1,2, \ldots, r$, and

$$
\begin{aligned}
\sum_{l=1}^{r} \alpha_{l}= & \frac{1}{1+\varepsilon}+\frac{1}{\left(1+\varepsilon^{-1}\right)(1+\varepsilon)} \\
& +\cdots+\frac{1}{\left(1+\varepsilon^{-1}\right)^{r-2}(1+\varepsilon)}+\frac{1}{\left(1+\varepsilon^{-1}\right)^{r-1}}
\end{aligned}
$$




$$
\begin{aligned}
&= \frac{1 /(1+\varepsilon)-1 /(1+\varepsilon)\left(1+\varepsilon^{-1}\right)^{r-1}}{1-1 /\left(1+\varepsilon^{-1}\right)} \\
&+\frac{1}{\left(1+\varepsilon^{-1}\right)^{r-1}}=1 \\
&\left(\alpha_{1}+\alpha_{2}+\cdots+\alpha_{r-1} \text { is a geometric progression }\right) .
\end{aligned}
$$

Remark 4. By Lemmas 2 and 3, it is an obvious fact that

$$
\left(\sum_{l=1}^{r} \alpha_{l} A_{l}^{T}\right)\left(\sum_{l=1}^{r} \alpha_{l} A_{l}\right) \leq \sum_{l=1}^{r} \alpha_{l} A_{l}^{T} A_{l} .
$$

First, we discuss the stability of the nominal switched discrete-time system. For the nominal switched discrete-time system,

$$
x(k+1)=A_{l} x(k), \quad l=1,2, \ldots, r .
$$

Lemma 5. There exists a switching law for the nominal switched discrete-time system (9) such that the system (9) is asymptotically stable if there exist a symmetric matrix $P>0$ and positive constants $\alpha_{l}(1 \leq l \leq r)$ satisfying $\sum_{l=1}^{r} \alpha_{l}=1$ such that

$$
\sum_{l=1}^{r} \alpha_{l}\left(A_{l}^{T} P A_{l}\right)-P<0 .
$$

Proof. If there exist a symmetric matrix $P>0$ and positive constants $\alpha_{l}(1 \leq l \leq r)$ satisfying $\sum_{l=1}^{r} \alpha_{l}=1$ such that the inequality (10) holds, the inequality (10) is equivalent to the following inequality:

$$
\sum_{l=1}^{r} \alpha_{l}\left(A_{l}^{T} P A_{l}-P\right)<0, \quad l=1,2, \ldots, r .
$$

Then, for all $x(k) \in \mathbf{R}^{n}, x(k) \neq 0$,

$$
x^{T}(k)\left[\sum_{l=1}^{r} \alpha_{l}\left(A_{l}^{T} P A_{l}-P\right)\right] x(k)<0 .
$$

Therefore, it follows that, for any $k$, at least there exists an $l \in$ $\{1,2, \ldots, r\}$ such that

$$
x^{T}(k)\left[A_{l}^{T} P A_{l}-P\right] x(k)<0 .
$$

From (13), it implies that a convex combination of the corresponding Lyapunov function (12) is negative along the trajectory, and from (13) at least one must be negative. Thus, the nominal switched discrete-time system (9) is asymptotically stable.

Lemma 6 (see [34]). Chebyshev inequality holds for any matrix $W_{i} \in R^{n \times n}$ :

$$
\left(\sum_{i=1}^{N} A_{i}\right)^{T}\left(\sum_{i=1}^{N} A_{i}\right) \leq N\left(\sum_{i=1}^{N} A_{i}^{T} A_{i}\right) .
$$

In the study of switched large-scale discrete-time systems, the main problems are how to constructively design a switching rule and how to derive sufficient stability conditions which can guarantee the stability of the switched large-scale discrete-time systems under the switching rule.

\section{Stabilization Analysis}

For the switched large-scale discrete-time system (2) with $r$ individual systems and each individual system composed of $N$ interconnected subsystems, assume that $\sum_{l=1}^{r} \sqrt{2} \alpha_{l}^{1 / 2} A_{l_{i i}}$ are Schur matrices. To investigate the stability of system (1), we denote $x^{T}(k)=\left[\begin{array}{llll}x_{1}^{T} & x_{2}^{T} & \cdots & x_{N}^{T}\end{array}\right]^{T}$ and $P=$ $\operatorname{diag}\left\{P_{1}, P_{2}, \ldots, P_{N}\right\}$, where

$$
2\left(\sum_{l=1}^{r} \alpha_{l}^{1 / 2} A_{l_{i i}}\right)^{T} P_{i}\left(\sum_{l=1}^{r} \alpha_{l}^{1 / 2} A_{l_{i i}}\right)-P_{i}=-I .
$$

Theorem 7. Consider the switched large-scale discrete-time system (1), and assume that $\sum_{l=1}^{r} \alpha_{l}^{1 / 2} A_{l_{i i}}$ are Schur matrices. There exists a switching law such that the switched large-scale discrete-time system (1) is asymptotically stable, if matrices $P_{1}, P_{2}, \ldots, P_{N}>0$ satisfy (15) and the following inequality (16) holds:

$$
\sum_{l=1}^{r} \sum_{\substack{j \neq i \\ j=1}}^{N} \alpha_{l}\left\|A_{l_{j i}}^{T} P_{j} A_{l_{j i}}\right\| \leq 0.5(N-1)^{-1},
$$

where $\alpha_{l}=1 /\left(1+\varepsilon^{-1}\right)^{l-1}(1+\varepsilon),(l=1,2, \ldots, r-1), \alpha_{r}=$ $1 /\left(1+\varepsilon^{-1}\right)^{r-1}$ and $\varepsilon>0$.

Proof. By inequality (12), we obtain

$$
\begin{gathered}
\sum_{i=1}^{N}\left\{\left[\sum_{l=1}^{r} \alpha_{l}\left(A_{l_{i i}} x_{i}(k)+\sum_{\substack{j \neq i \\
j=1}}^{N} A_{l_{i j}} x_{j}(k)\right)^{T}\right.\right. \\
\left.\quad \times P_{i}\left(A_{l_{i i}} x_{i}(k)+\sum_{\substack{j \neq i \\
j=1}}^{N} A_{l_{i j}} x_{j}(k)\right)\right] \\
\left.-x_{i}^{T}(k) P_{i} x_{i}(k)\right\} \\
\leq \sum_{i=1}^{N}\left\{\begin{array}{l}
\sum_{l=1}^{r} \alpha_{l}\left(A_{l_{i i}} x_{i}(k)\right)^{T} P_{i}\left(A_{l_{i i}} x_{i}(k)\right) \\
+\sum_{l=1}^{r} \alpha_{l}\left(A_{l_{i i}} x_{i}(k)\right)^{T} P_{i}\left(\sum_{j \neq i}^{N} A_{l_{i j}} x_{j}(k)\right.
\end{array}\right)
\end{gathered}
$$




$$
\begin{aligned}
& +\sum_{l=1}^{r} \alpha_{l}\left(\sum_{\substack{j \neq i \\
j=1}}^{N} A_{l_{i j}} x_{j}(k)\right)^{T} P_{i}\left(A_{l_{i i}} x_{i}(k)\right) \\
& +\sum_{l=1}^{r} \alpha_{l}\left(\sum_{\substack{j \neq i \\
j=1}}^{N} A_{l_{i j}} x_{j}(k)\right)^{T} P_{i}\left(\sum_{\substack{j \neq i \\
j=1}}^{N} A_{l_{i j}} x_{j}(k)\right) \\
& \left.-x_{i}^{T}(k) P_{i} x_{i}(k)\right\} \\
& \leq \sum_{i=1}^{N}\left\{\sum _ { l = 1 } ^ { r } \alpha _ { l } \left[2\left(A_{l_{i i}}(k)\right)^{T} P_{i}\left(A_{l_{i i}} x_{i}(k)\right)\right.\right. \\
& +\sum_{l=1}^{r} 2 \alpha_{l}\left(\sum_{\substack{j \neq i \\
j=1}}^{N} A_{l_{i j}} x_{j}(k)\right)^{T} \\
& \left.\times P_{i}\left(\sum_{\substack{j \neq i \\
j=1}}^{N} A_{l_{i j}} x_{j}(k)\right)\right] \\
& \left.-x_{i}^{T}(k) P_{i} x_{i}(k)\right\} \\
& \leq \sum_{i=1}^{N}\left\{\sum_{l=1}^{r} 2 \alpha_{l}\left(A_{l_{i i}} x_{i}(k)\right)^{T} P_{i}\left(A_{l_{i i}} x_{i}(k)\right)\right. \\
& +\sum_{l=1}^{r} 2 \alpha_{l}(N-1) \\
& \times\left(\sum_{\substack{j \neq i \\
j=1}}^{N}\left(A_{l_{i j}} x_{j}(k)\right)^{T} P_{i}\left(A_{l_{i j}} x_{j}(k)\right)\right) \\
& \left.-x_{i}^{T}(k) P_{i} x_{i}(k)\right\} \\
& \leq \sum_{i=1}^{N} x_{i}^{T}(k)\{-I+2(N-1) \\
& \left.\times\left(\sum_{\substack{l=1 \\
j \neq i}}^{r} \sum_{\substack{j \neq 1 \\
j}}^{N}\left\|A_{l_{j i}}^{T} P_{j} A_{l_{j i}}\right\|\right)\right\} x_{i}(k) .
\end{aligned}
$$

If conditions (16) are satisfied, then the inequality (17) is smaller than zero for $i=1,2, \ldots, N$ and $l=1,2, \ldots, r$. Therefore, the switched large-scale discrete-time system is asymptotically stable.

Remark 8. For the switched large-scale discrete-time system (1) with arbitrary $r$ individual systems and $N$ state vectors, Theorem 7 can be applied to cases when all individual subsystems are unstable. The particular method also can be applied to cases when $r>N$, or $r=N, r<N$.

Switching Law. Switched large-scale discrete-time system (1) is switched to or stays at mode $l$ at sampling step $k$ if (18) is satisfied at $k$ :

$$
\begin{aligned}
& \sum_{i=1}^{N}\left\{\left[A_{l_{i i}} x_{i}(k)+\sum_{\substack{j \neq i \\
j=1}}^{N} A_{l_{i j}} x_{j}(k)\right]^{T}\right. \\
& \left.\quad \times P_{i}\left[A_{l_{i i}} x_{i}(k)+\sum_{\substack{j \neq i \\
j=1}}^{N} A_{l_{i j}} x_{j}(k)\right]-P_{i}\right\}<0, \\
& l=1,2, \ldots, r .
\end{aligned}
$$

\section{Conclusion}

We have adopted the Lyapunov stability theorem to deal with the stabilization analysis of switched large-scale discretetime systems. The results are straightforward. By a simple switching law, the sufficient stability conditions have been derived for the switched large-scale discrete-time systems via the state-driven switching. The results can be applied to cases when $r>N, r=N$, or $r<N$ for the switched large-scale discrete-time system with arbitrary $r$ individual systems and $N$ state vectors. In addition, the particular method can be applied to cases when all individual subsystems are unstable.

\section{Acknowledgment}

This work is supported by the National Science Council, Taiwan, under Grants nos. NSC101-2221-E-218-027 and NSC1002632-E-218-001-MY3.

\section{References}

[1] D. Liberzon and A. S. Morse, "Basic problems in stability and design of switched systems," IEEE Control Systems Magazine, vol. 19, no. 5, pp. 59-70, 1999.

[2] A. S. Morse, "Supervisory control of families of linear set-point controllers. I. Exact matching," IEEE Transactions on Automatic Control, vol. 41, no. 10, pp. 1413-1431, 1996.

[3] R. Frasca, L. Iannelli, and F. Vasca, "Dithered sliding-mode control for switched systems," IEEE Transactions on Circuits and Systems II, vol. 53, no. 9, pp. 872-876, 2006.

[4] G. Zhai, D. Liu, J. Imae, and T. Kobayashi, "Lie algebraic stability analysis for switched systems with continuous-time 
and discrete-time subsystems," IEEE Transactions on Circuits and Systems II, vol. 53, no. 2, pp. 152-156, 2006.

[5] T.-H. Kim, S.-H. Lee, and J.-T. Lim, "Stability analysis of switched systems via redundancy factors," International Journal of Systems Science, vol. 32, no. 10, pp. 1199-1204, 2001.

[6] X. Zhang, Z. Q. Xia, and Y. Gao, "Exponential stabilizability of switched systems with polytopic uncertainties," Journal of Applied Mathematics, vol. 2012, Article ID 853170, 15 pages, 2012.

[7] B. Du and X. Zhang, "Delay-dependent stability analysis and synthesis for uncertain impulsive switched system with mixed delays," Discrete Dynamics in Nature and Society, vol. 2011, Article ID 381571, 9 pages, 2011.

[8] Z. Ji, L. Wang, and G. Xie, "Quadratic stabilization of switched systems," International Journal of Systems Science, vol. 36, no. 7, pp. 395-404, 2005.

[9] D. Cheng, L. Guo, and J. Huang, "On quadratic Lyapunov functions," IEEE Transactions on Automatic Control, vol. 48, no. 5, pp. 885-890, 2003.

[10] S. Yang, X. Zhengrong, C. Qingwei, and H. Weili, "Robust reliable control of switched uncertain systems with timevarying delay," International Journal of Systems Science, vol. 37, no. 15, pp. 1077-1087, 2006.

[11] Z. G. Li, C. Y. Wen, and Y. C. Soh, "Stabilization of a class of switched systems via designing switching laws," IEEE Transactions on Automatic Control, vol. 46, no. 4, pp. 665-670, 2001.

[12] Z. Sun and S. S. Ge, "Analysis and synthesis of switched linear control systems," Automatica, vol. 41, no. 2, pp. 181-195, 2005.

[13] Z. Ji, L. Wang, G. Xie, and F. Hao, "Linear matrix inequality approach to quadratic stabilisation of switched systems," IEE Proceedings, vol. 151, no. 3, pp. 289-294, 2004.

[14] J. P. Hespanha and A. S. Morse, "Stability of switched systems with average dwell-time," in Proceedings of the 38th IEEE Conference on Decision and Control (CDC '99), pp. 2655-2660, December 1999.

[15] G. Zhai, B. Hu, K. Yasuda, and A. N. Michel, "Stability analysis of switched systems with stable and unstable subsystems: an average dwell time approach," International Journal of Systems Science, vol. 32, no. 8, pp. 1055-1061, 2001.

[16] B. Lu and F. Wu, "Switching LPV control designs using multiple parameter-dependent Lyapunov functions," Automatica, vol. 40, no. 11, pp. 1973-1980, 2004.

[17] S.-H. Lee, T.-H. Kim, and J.-T. Lim, "A new stability analysis of switched systems," Automatica, vol. 36, no. 6, pp. 917-922, 2000.

[18] Z. Ji and L. Wang, "Quadratic stabilization of uncertain discretetime switched linear systems," in Proceedings of the IEEE International Conference on Systems, Man and Cybernetics (SMC '04), pp. 1492-1497, October 2004.

[19] G. Xie and L. Wang, "Stabilization of a class of hybrid discretetime systems," in Proceedings of the 2003 IEEE Conference on Control Applications, pp. 1404-1409, June 2003.

[20] G. Zhai, H. Lin, A. N. Michel, and K. Yasuda, "Stability analysis for switched systems with continuous-time and discrete-time subsystems," in Proceedings of the American Control Conference (AAC '04), pp. 4555-4560, July 2004.

[21] J. Daafouz, P. Riedinger, and C. Iung, "Stability analysis and control synthesis for switched systems: a switched Lyapunov function approach," IEEE Transactions on Automatic Control, vol. 47, no. 11, pp. 1883-1887, 2002.

[22] D. Xie, L. Wang, F. Hao, and G. Xie, "LMI approach to $L_{2}$-gain analysis and control synthesis of uncertain switched systems," IEE Proceedings, vol. 151, no. 1, pp. 21-28, 2004.
[23] D. Xie, L. Wang, F. Hao, and G. Xie, "Robust stability analysis and control synthesis for discrete-time uncertain switched systems," in Proceedings of the 42nd IEEE Conference on Decision and Control, pp. 4812-4817, December 2003.

[24] Z. Sun, "Sampling and control of switched linear systems," Journal of the Franklin Institute, vol. 341, no. 7, pp. 657-674, 2004.

[25] M. S. Mahmoud, M. F. Hassan, and M. G. Darwish, Large-Scale Control Systems, Marcel Dekker, New York, NY, USA, 1985.

[26] M. Y. Waziri and Z. A. Majid, "An improved diagonal Jacobian approximation via a new Quasi-Cauchy condition for solving large-scale systems of nonlinear equations," Journal of Applied Mathematics, vol. 2013, Article ID 875935, 6 pages, 2013.

[27] B. Labibi, "Decentralized control via disturbance attenuation and eigenstructure assignment," IEEE Transactions on Circuits and Systems II, vol. 53, no. 6, pp. 468-472, 2006.

[28] S. Xie and L. Xie, "Stabilization of a class of uncertain large-scale stochastic systems with time delays," Automatica, vol. 36, no. 1, pp. 161-167, 2000.

[29] W.-J. Wang and L.-G. Mau, "Stabilization and estimation for perturbed discrete time-delay large-scale systems," IEEE Transactions on Automatic Control, vol. 42, no. 9, pp. 1277-1282, 1997.

[30] S. Xie and L. Xie, "Decentralized stabilization of a class of interconnected stochastic nonlinear systems," IEEE Transactions on Automatic Control, vol. 45, no. 1, pp. 132-137, 2000.

[31] S. Xie, L. Xie, Y. Wang, and G. Guo, "Decentralized control of multimachine power systems with guaranteed performance," IEE Proceedings, vol. 147, no. 3, pp. 355-365, 2000.

[32] $\mathrm{H}$. Wu, "Decentralized adaptive robust control for a class of large-scale systems including delayed state perturbations in the interconnections," IEEE Transactions on Automatic Control, vol. 47, no. 10, pp. 1745-1751, 2002.

[33] W. J. Wang and L. Luoh, "Stability and stabilization of fuzzy large-scale systems," IEEE Transactions on Fuzzy Systems, vol. 12, no. 3, pp. 309-315, 2004.

[34] K. Zhou and P. P. Khargonekar, "Robust stabilization of linear systems with norm-bounded time-varying uncertainty," Systems and Control Letters, vol. 10, no. 1, pp. 17-20, 1988. 


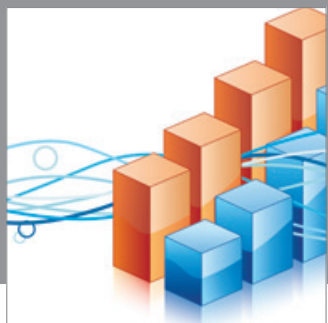

Advances in

Operations Research

mansans

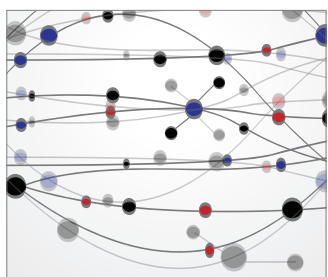

The Scientific World Journal
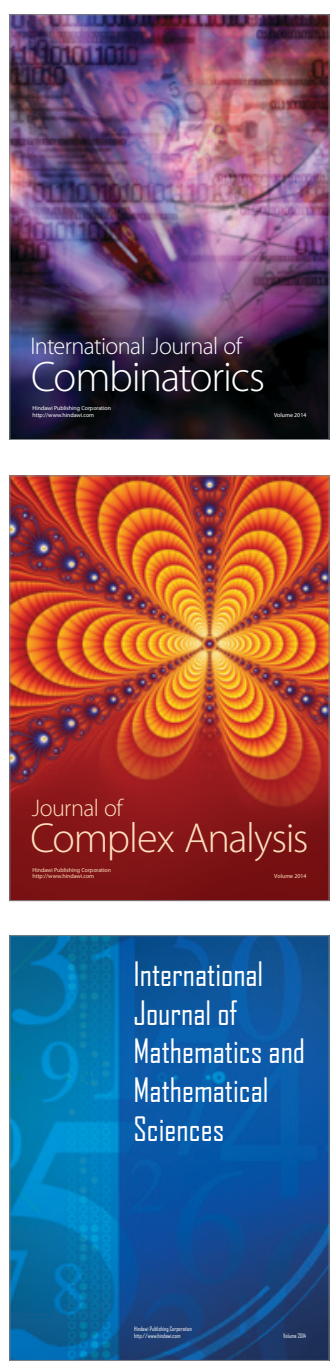
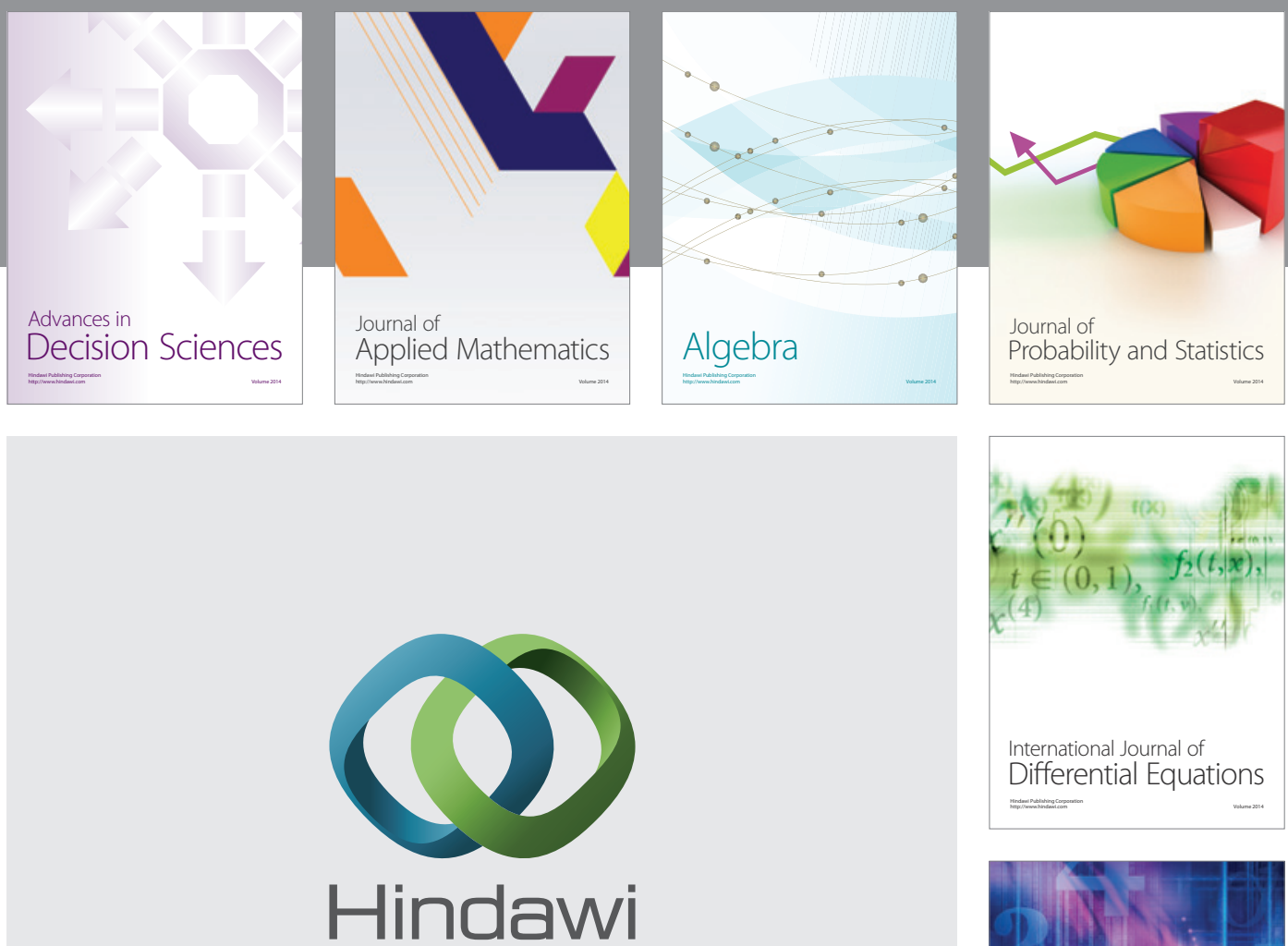

Submit your manuscripts at http://www.hindawi.com
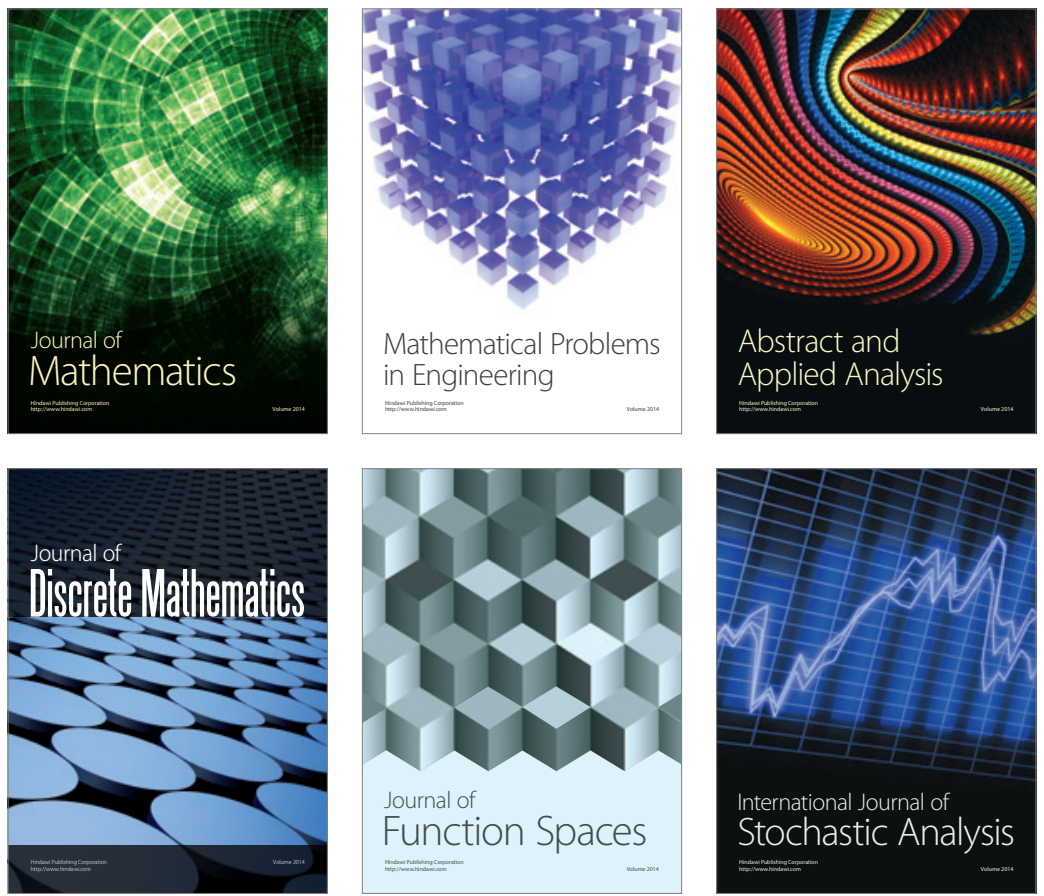

Journal of

Function Spaces

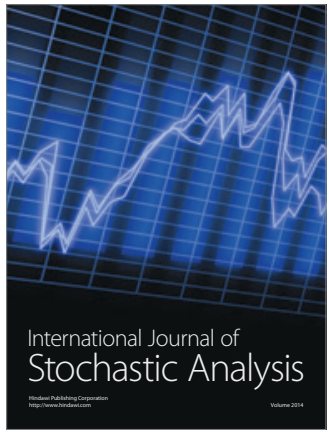

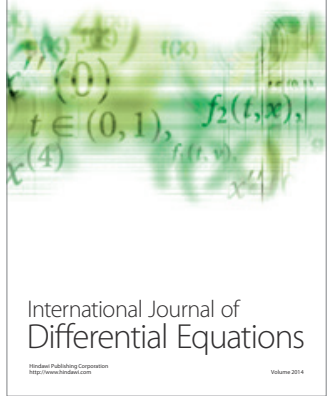
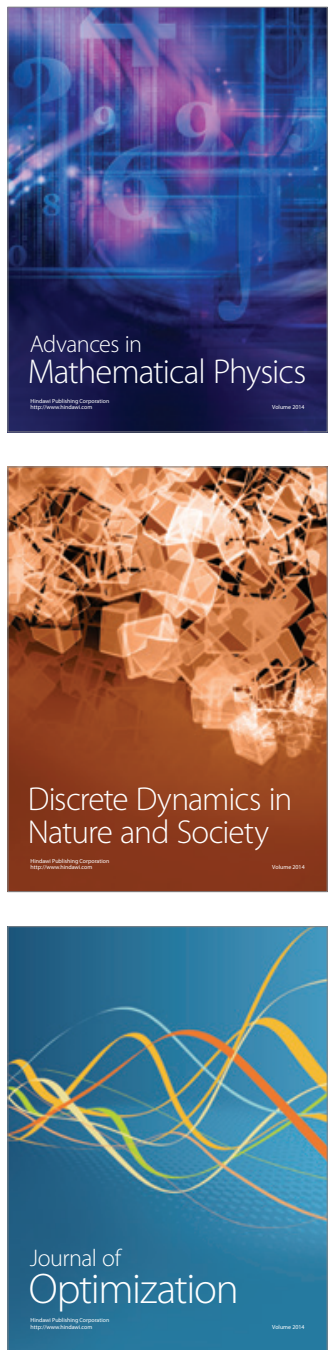